\title{
Infektionsprävention oder Hygiene?
}

\author{
Heinz-Michael Just
}

Wenn in deutschen Krankenhäusern über Hygiene diskutiert wird, drehte es sich in der Vergangenheit meist um bauliche Aspekte wie Schleusen zum Abgrenzen „sensibler“ Bereiche, getrennt für Patienten, Personal und Waren mit ebenfalls streng getrennter „Wegeführung“ für „infektiöse“ und „nicht-infektiöse“ Patienten und für die Abfälle. Auch heute geht es oft immer noch primär um „reine“ und „unreine“ Arbeitsräume, um raumlufttechnische Anlagen (RLT) mit „geeigneten“ Luftfiltern für eine möglichst keimfreie Umgebungsluft oder um Maßnahmen zur Sanierung von Wasserleitungsnetzen, zumindest aber den Einbau von Wasserfiltern, für ein Wasser frei von Legionellen und Pseudomonaden. Wo aber erscheint der Patient, wo seine Infektionen?

Mit Inkrafttreten des Infektionsschutzgesetz (IfSG, 2001) wurde die „Surveillance“ eingeführt, die Pflicht zur Erfassung und Bewertung (!) von nosokomialen Infektionen mit der Intention, durch ein verstärktes Problembewusstsein Infektionen zu verhindern, wobei konkrete Risiken benannt werden (invasive Maßnahmen, Fremdkörper). Parallel dazu (ebenfalls 2001) erschien die RKI-Empfehlung „Anforderung an die Hygiene bei der Aufbereitung von Medizinprodukten“. Diese konkretisiert die Maßnahmen zur Umsetzung der Vorgaben des Medizinproduktegesetzes und der Medizinproduktebetreiberverordnung für die Aufbereitung von Medizinprodukten zwischen Anwendungen an Patienten. Mit beiden gesetzlichen Anforderungen rückt der Patient in den Mittelpunkt mit Bezug zu nachgewiesenermaßen konkreten Infektionsgefährdungen. Wie also entstehen Infektionen und wie können Übertragungen verhindert werden?

Erstmals wird im IfSG ( $§ 23$ [2]) eine unabhängige Expertenkommission verbindlich eingesetzt, die „Empfehlungen zur Prävention nosokomialer Infektionen sowie zu betrieblich-organisatorischen und baulichfunktionellen Maßnahmen der Hygiene in Krankenhäusern und anderen medizinischen Einrichtungen“ nach wissenschaftlicher Evidenz kategorisiert erstellt. Diese Empfehlungen sollen wiederum „als Grundlage für die Erfüllung der Aufgaben des öffentlichen Gesundheitsdienstes im Rahmen seiner Überwachungsund Beratungsmaßnahmen“ dienen [1]. Damit wird für die Empfehlungen ein Niveau an Qualität und Transparenz gefordert, dem weder die alten (nicht evidenzbasierten) Empfehlungen von BGA/RKI noch viele der konkurrierenden deutschsprachigen Empfehlungen/Leitlinien verschiedenster Fachgesellschaften in dieser Form gerecht werden. War die Vielfalt an unterschiedlichen, sich manchmal gar widersprechender „Hygiene“-Empfehlungen für den Betroffenen in medizinischen Einrichtungen in der Vergangenheit mehr verwirrend als hilfreich, so stellt sich nach Inkrafttreten des IfSG erst recht die Frage nach dem Sinn solcher zusätzlicher Veröffentlichungen. Insbesondere in den Teilbereichen Lüftungstechnik und Isolierungsmaßnahmen wird dieses Dilemma aktuell und vielfältig deutlich.

Beiträge von R. Külpmann und R. Meierhans bzw. von I. Kappstein in den vorangegangenen wie auch in diesem Heft von Krankenhaushygiene up2date haben dargelegt, worin der Nutzen einer RLT-(raumlufttechnischen) Anlage besteht und was sie eben nicht zu leisten vermag. Es gibt in der Literatur mehr Hinweise darauf, dass postoperative Wundinfektionen durch RLT-Anlagen verursacht, als dass sie durch diese verhindert werden. Bau, Unterhalt und Wartung von RLTAnlagen sind kostenintensiv; eine unterlassene regelmäßige Wartung aber ggf. Ursache nosokomialer Infektionen. Aus den Beiträgen wird zudem deutlich, dass die Luft als Erregerreservoir für postoperative Wundinfektionen im Vergleich zum Verhalten des Operationsteams von untergeordneter Bedeutung ist. Mikrobiologische Untersuchungen der Fußböden, Wände und anderer Oberflächen in OP-Einheiten zeigen regelmäßig ein völlig anderes Keimspektrum als die Erregerspektren bei postoperativen Wundinfektionen. Deshalb sind Abklatschuntersuchungen in OPs, wie sie mancherorts immer noch bei Begehungen durch den ÖGD stattfinden und gar jüngst als Ausdruck der Ergebnisqualität bewertet wurden, weder gerechtfertigt noch evidenzbasiert [2]. Auch die aus 
dem Jahre 2005 stammenden Entwürfe des VDI (Verein deutscher Ingenieure) wie auch zur Neufassung der DIN 1946 Teil 4 (Stand 2005) stellen völlig abwegige und durch keine wissenschaftlichen Daten zu rechtfertigende Forderungen an Raumklassen und deren Zuordnung zu invasiven Maßnahmen auf. Diese würden lediglich zu immensen unsinnigen Kosten führen, eine Sichtweise, die auch in einer Reaktion der Bauministerkonferenz offiziell geäußert wurde.

Dies zeigt, wie wichtig es ist, dass sich die Verantwortlichen aller beteiligten Berufsgruppen ihrer Pflicht bewusst sind, Empfehlungen und Forderungen auf ihre Berechtigung, d. h. ihre Evidenz hin zu überprüfen. Evidenz meint international aber wissenschaftlich gesicherte Erkenntnisse und nicht noch so einmütig erzielte Konsensusbeschlüsse - unabhängig davon, wie wissenschaftlich angesehen evtl. dahinter stehende Fachgesellschaften auch sein mögen! So sind beispielsweise bei Verlautbarungen des DIN (Deutsches Institut für Normung e.V.) nicht selten Lobbyinteressen aufgrund eines hohen Anteils an Industrievertretern unter den „Experten“ deutlich erkennbar.

Medizinische Einrichtungen haben sich in der Vergangenheit in der Regel an Empfehlungen der „Hygiene“ orientiert, ohne deren Schlüssigkeit zu hinterfragen.

Das Ergebnis war oft ein standardisiertes Vorgehen mit gleichen Regeln für alle, siehe der Umgang mit multiresistenten Keimen. Hier wird meist eine strikte Isolierung auch für nur besiedelte Patienten gefordert, womit automatisch die Unterbringung in einem Einzelzimmer gemeint ist. Untersuchungen hierzu konnten bislang nur belegen, dass solche Patienten dann ärztlich schlechter versorgt und pflegerisch schlechter betreut werden [3]. Der Beweis für eine Verhinderung von Keimübertragungen allein durch die räumliche Isolierung steht aus. In Studien, die dies zu belegen meinen, kam immer ein ganzes Paket sogenannter „Barrieremaßnahmen“ zur Anwendung, unter denen auch solche waren, die eine Weiterverbreitung verhindert haben. Sehr zeit- und kostenintensiv ist hierbei das rituelle Umkleiden vor jedem Patientenkontakt.

In dem Beitrag von H. Rüden et al. in diesem Heft wird auf oft kontrovers diskutierte Aspekte diverser Klei- dervorschriften eingegangen. Insbesondere wird deutlich gemacht, was den Sinn einer Arbeitskleidung im Unterschied zu einer Schutzkleidung ausmacht. Letztere ist arbeitsmedizinisch klar definiert; diese Definition trifft aber im Zusammenhang mit dem Einsatz bei sogenannten ,infektiösen“ Patienten immer seltener zu. Die Schutzkleidung im Sinne der Arbeitsmedizin soll nur den Träger vor einer persönlichen Gefährdung schützen, während die infektionsbedingte Schutzkleidung im Klinikalltag meist eine Weiterverbreitung von Keimen auf andere, aufgrund ihrer Erkrankung gefährdete Patienten verhindern soll. Neben dem klassischen Übertragungsweg ist das jeweilige tätigkeitsspezifische Risiko einer Keimübertragung entscheidend, was verdeutlicht, warum es wenig sinnvoll ist, ein starres Maßnahmenpaket ausschließlich mit dem nachgewiesenen Mikroorganismus zu verknüpfen und die jeweilige Situation bzw. Tätigkeit außer Acht zu lassen.

All dies verdeutlicht die gestiegenen Anforderungen an eine effiziente, moderne Infektionsprävention, die mit klassischen „Hygieneempfehlungen“ mit Schwerpunkt Desinfektion und Isolierung nur noch wenig gemein hat.

Eine moderne Infektionsprävention umfasst neben einer frühzeitigen, gezielten mikrobiologischen Diagnostik einen verantwortungsvolleren Einsatz von Antibiotika, gefolgt von konsequentem Befolgen evidenzbasierter situationsangepasster Hygienemaßnahmen durch alle Betroffenen. Solche Hygienemaßnahmen orientieren sich an evidenzbasierten Empfehlungen nationaler wie internationaler Richtlinien, unter Berücksichtigung erregerspezifischer Aspekte wie Pathogenität und Virulenz, des Übertragunsweges und epidemiologischer Eigenschaften. Dies und die genannten gesetzlichen Verpflichtungen erfordern deutlich besser ausgebildetes Hygienepersonal in allen Berufsgruppen und allen medizinischen Einrichtungen $[4,5]$.

Die Politik hat unserem Gesundheitswesen einen Sparzwang verordnet, der sich nicht an den medizinischen Notwendigkeiten orientiert und in dessen Folge die Qualität der medizinischen Versorgung allenfalls in Wahlreden von Politikern noch an erster Stelle stehen dürfte. 


\section{Literatur}

1 Bales S, Baumann HG, Schnitzler N. Infektionsschutzgesetz - Kommentar und Vorschriftensammlung. 2. Aufl. Stuttgart: Kohlhammer, 2003: $197-198$

2 Billing J, Fembacher L, Schweitzer S. Erhebungen zur Struktur-, Prozess- und Ergebnisqualität in Einrichtungen für Ambulantes Operieren - ein Erfahrungsbericht. Hyg Med 2006; 31: 385 - 394
3 Stelfox HAT, Bates DW, Redelmeier DA. Safety of patients isolated for infection control. JAMA 2003; 290: $1899-1905$

4 Health Canada, Nocosomial and Occupational Infections Section. Development of a resource model for infection prevention and control programs in acute, long term, and home care settings: Conference proceedings of the Infection Prevention and Control Alliance. Am J Infect Control 2004; 32: 2 -6

5 Van den Broek PJ, Kluytmans JAJW et al. How many infection control staff do we need in hospitals? J Hosp Inf 2007; 65: 108 -111 